\title{
PERSPECTIVE
}

\section{Sports and Exercise Medicine and the evolution of musculoskeletal ultrasonography}

\author{
Dinesh Sirisena ${ }^{1}$, Mandy Zhang $^{2}$, Shauna Sim ${ }^{1}$
}

\begin{abstract}
Musculoskeletal Ultrasound is likened to the traditional stethoscope for Sports Medicine physicians. This article considers key features of ultrasonography when it is applied in the musculoskeletal setting, important steps when developing this skill, and also the perspective from and trainee learning this skill in Singapore. In essence, it requires a basic understanding of the physics, knowledge of sonoanotomy and most importantly suitable mentorship to steer and guide learning.
\end{abstract}

Keywords: Sports Medicine, Musculoskeletal, Ultrasonography

\section{Introduction}

Sports and Exercise Medicine (SEM) remains a young speciality that is exciting and constantly evolving. Since its inception, the skill base and areas of practice have expanded to encompass expertise in musculoskeletal, exercise and team medicine, ultrasound imaging and non-surgical interventions such as shockwave therapy and ultrasound-guided procedures. With such a diverse range of skills, it has required SEM clinicians to gain additional training to better equip themselves to help their patients. Musculoskeletal ultrasound is certainly one of these areas and it is now the expectation from many patients that their SEM specialist will be able to provide this modality of investigation to complement their clinical assessment.

${ }^{1}$ Sports Medicine Centre, Khoo Teck Puat Hospital, Singapore, ${ }^{2}$ Sports Medicine Centre, Changi General Hospital, Singapore.

\section{Correspondence:}

Email: dinesh.sirisena@ktph.com.sg

\section{Ultrasound in SEM - the 'Sports Physician's Stethoscope'}

Ultrasound is a valuable diagnostic tool that has gained popularity among SEM clinicians, often dubbed as the 'sports physician's stethoscope'. Indeed, bedside ultrasound imaging allows immediate correlation with patients' symptoms and provides important information to the physician. Using an anatomic structural approach, ultrasound is applied to visualize soft tissues such as ligaments, tendons, bursa and muscles. Common clinical indications would include rotator cuff disease, tennis elbow, golfer's elbow, patellar tendinopathy and plantar fasciitis, among many others.

Several properties of musculoskeletal ultrasound are important to the specialty of sports medicine. These include real-time imaging, split-screen presentation comparing the symptomatic and asymptomatic side, and panoramic view. Other applications include colour-doppler and sonoelastography. The 
benefit of real-time imaging is that it allows for stress and dynamic maneuvers, while joints move through their expected range of motion. This enhances the diagnostic quality of ultrasound, especially when the pathology is not revealed by a static evaluation. Examples include resisted ankle dorsiflexion and external rotation to evaluate subluxation of peroneal tendons due to torn retinaculum [1] and passive or active shoulder abduction to visualize sub acromial-sub deltoid bursal thickening, bunching of the supraspinatus tendon laterally and superior migration of the humeral head in sub acromial impingement [2]. Sonography with colour-doppler is a useful tool to determine the activity or recurrence of disease, as in the case of synovial inflammation, abnormal connective tissue or inflammatory reaction. This technique also allows for detection of blood vessels to provide a safe needle trajectory during an injection procedure. Another area of avid interest involves the musculoskeletal applications of sonoelastography; based on the principle that the application of pressure induces tissue deformation and can be used to assess the mechanical properties of soft tissue qualitatively and quantitatively. Altered tissue elasticity often occur with degeneration or pathological changes. Sonoelastography shows early promise in improved diagnostic accuracy, prediction of at-risk tendons and prognostication capability beyond conventional grey-scale ultrasound.

\section{Developing musculoskeletal ultrasound skills}

At this juncture, it is important to re-iterate that musculoskeletal ultrasound complements our clinical skills and does not replace them. Often in specialist or tertiary centres, there is the temptation to deviate from usual clinical assessment and gravitate to this investigation.
However, over-dependency on this modality risks de-skilling clinicians from the role for which they were trained, namely, the clinical assessment, counselling and treatment of patients. Nevertheless, SEM practitioners have excelled at the integration of musculoskeletal ultrasound in clinical practice and are often helping to re-write the boundaries in terms of investigative and interventional practice, particularly as we have the benefit of getting to know our patients and cultivating the doctorpatient relationship.

The key to developing this skill is to consider ultrasound imaging and interventions as a longitudinal skill, that requires initial guidance and mentorship, followed by regular appraisal and self-reflection. In the initial phases, correct instruction on the functions of an ultrasound machine, controls and applications is necessary to understand how to obtain an image and optimise it. In addition, there is the correct positioning of patients and how to manage perturbations in body habitus and anatomy, to know that the area being scanned is indeed the area of interest. A simple example of this is in the shoulder, where some patients have a more laterally placed bicipital groove than others; with training, the clinician will know how to position the patient and being the study, but with experience they will be able to appreciate the subtle variations that are possible and know what to do when these are encountered.

In the next phase, once normal ultrasound imaging is appreciated, there is the recognition of abnormal and what might be the pathological process accounting for the patient's symptoms. Often, this phase requires regular contact with a supervisor or mentor, evaluation and re-evaluation of images obtained and the acceptance that being able to 
identify these nuances takes scanning time. Finally, when scanning becomes habitual and anatomy or pathological changes can be recognised, then interventions can be considered; the commonest one that is taught is the sub-acromial bursa injection (Figure 1). Although this can be routinely done without ultrasound guidance, it is well known that adding guidance can improve delivery of the medication and hopefully patient outcomes [3, 4]. Requiring time, supervision and selfreflection on how to improve technique, developing competency with this procedure can then lead on to further ones such as highvolume, hydrodilatation, tendon stripping, platelet rich plasma, prolotherapy and even spine injections.

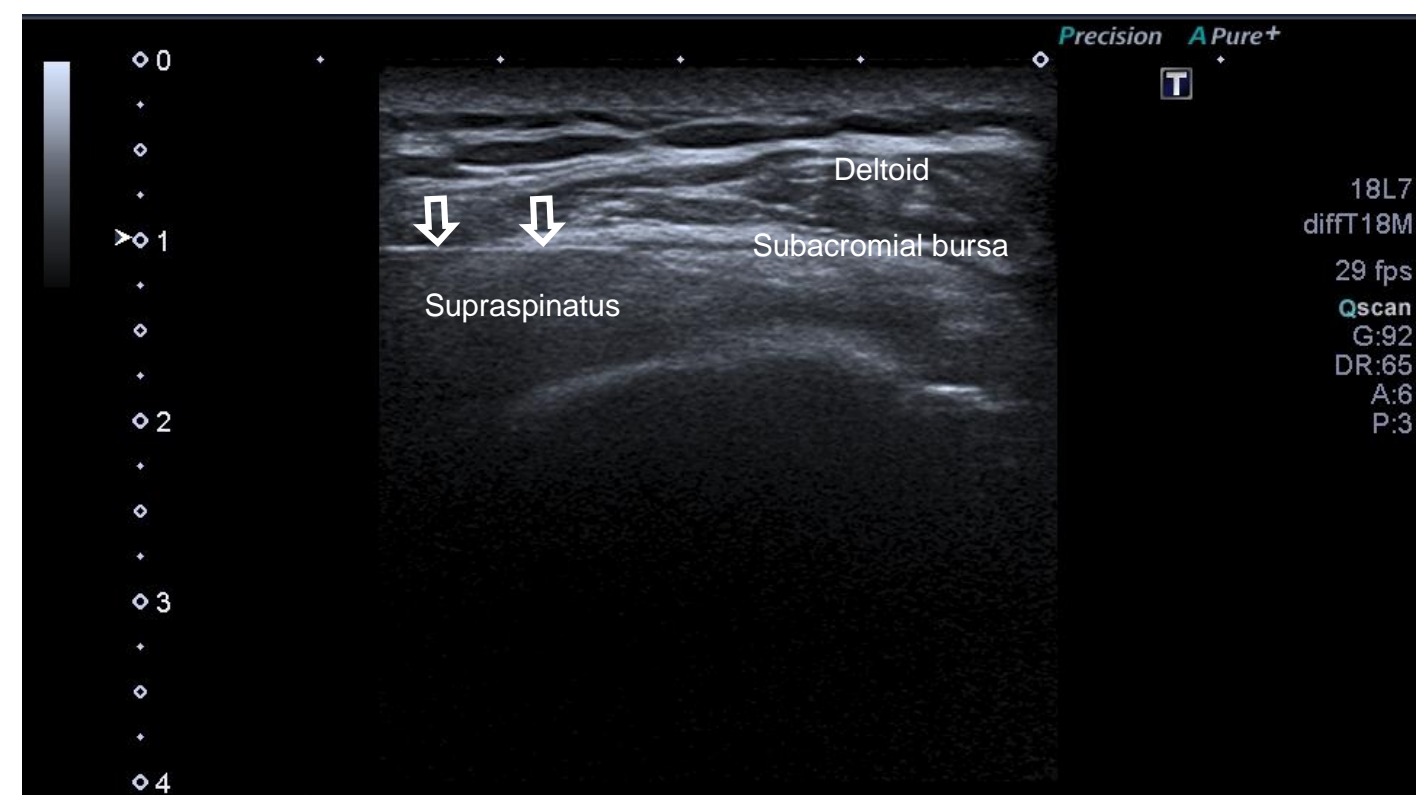

Figure 1. The subacromial, sub deltoid bursa as seen on ultrasound. The needle can be seen entering the bursa (indicated by the $\downarrow$ ).

\section{Ultrasound Training (a Sports Medicine Trainee's Perspective)}

I started formal training as a Sports Medicine registrar in 2019, however prior to this I had undertaken some attachments at the Sports Medicine Centre at Khoo Teck Puat Hospital, (KTPH) Singapore and was provided some exposure to ultrasound training.

The key challenge I have faced is that formal training in ultrasound imaging and interventions, is not widely available as a Sports Medicine trainee and most of the teaching I had was provided by the Consultants in clinic during my attachment. To my knowledge, there is only one ultrasound course (https://www.aaces.sg/musculoskeletalultrasou nd) in Singapore, held annually by Sports Medicine physicians. Although other musculoskeletal ultrasound courses are available, these are tailored to radiology trainees/radiographers and do not focus on Sports Medicine conditions or incorporate a consultation/clinical aspect.

I overcame this by seeking out extra teaching from the Consultants and having them supervise my ultrasound imaging of patients in 
clinic. Although it would have been useful to be proficient in ultrasound scanning prior to starting my training, lack of opportunities to pick-up ultrasound outside the clinic setting was a limitation, but now, as a trainee who has had hands-on experience, I will be developing my competency in ultrasound-guided procedures. Again, with this, although I have attended various courses, some of which have included practical sessions, there is no comparison to supervised procedures on suitable patients.
With the growth of Sports Medicine and the training programs, I think it would be good to take steps to have more opportunities for formal training in ultrasound imaging and subsequently procedural skills. I aim to incorporate these skills into my clinical practice so that it can benefit my future patients.

\section{Conflicts of interest:}

Authors declare no conflicts of interest.

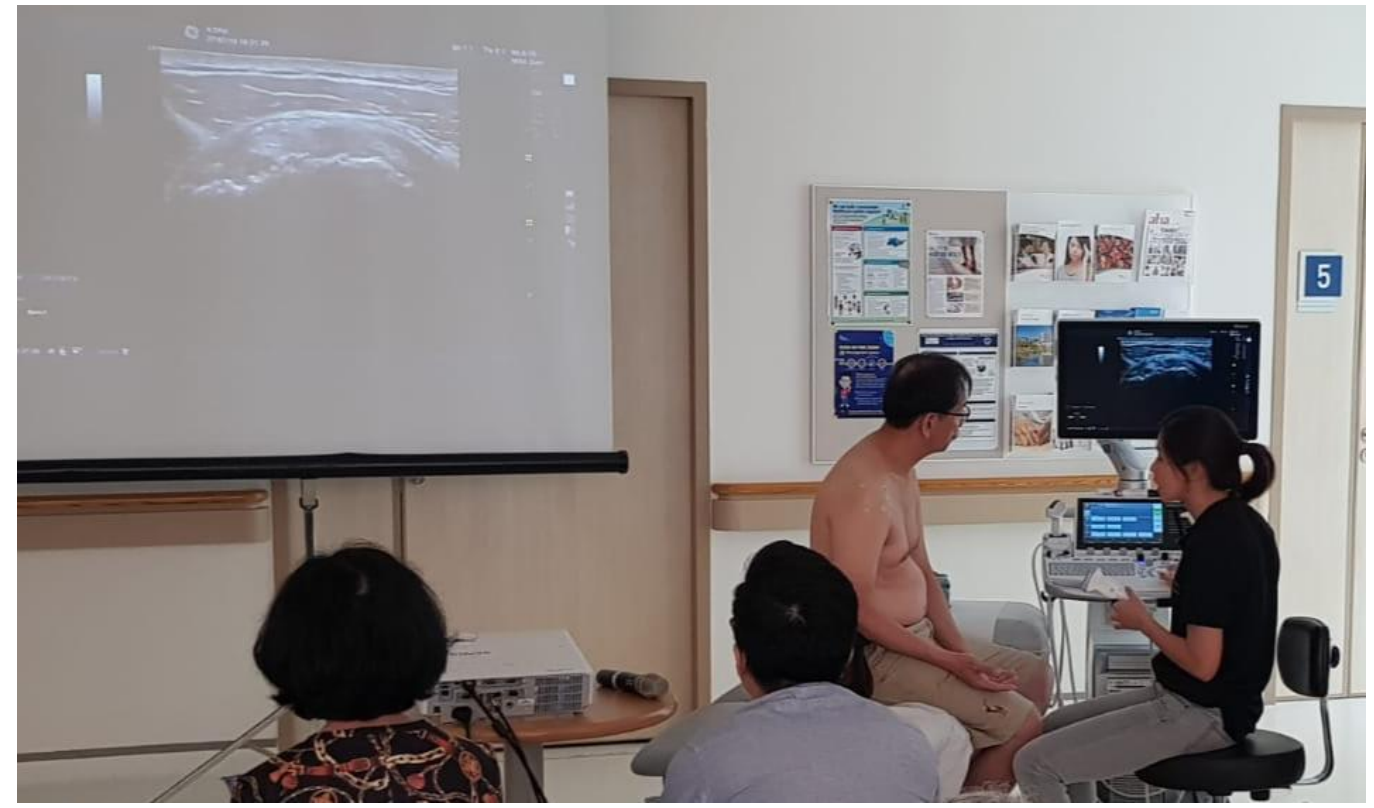

Figure 2. Dr Shauna Sim demonstrating ultrasound imaging of the shoulder at the GP Masterclass Series held at Khoo Teck Puat Hospital.

\section{References}

1. Neustadter, J., S.M. Raikin, and L.N. Nazarian, Dynamic Sonograpic Evaluation of Peroneal Tendon Subluxation. American Journal of Roentgenology, 2004. 183: p. 985-988.

2. .Park, J., et al., Dynamic ultrasonography of the shoulder. Ultrasonography, 2018. 37(3): p. 190-199.
3. Tagliafico, A., et al., Ultrasound-guided interventional procedures around the shoulder. Radiologia Medica, 2014. 119(5): p. 318-326.

4. Zufferey, P., et al., A controlled trial of the benefits of ultrasound-guided steroid injection for shoulder pain. Joint Bone Spine, 2012. 79(2): p. 166-169. 